Check for updates

Cite this: RSC Adv., 2019, 9, 2402

\title{
Efficient remediation of crude oil-contaminated soil using a solvent/surfactant system
}

\author{
Maoxin Wang, ${ }^{\text {a Bo Zhang, }}{ }^{\mathrm{b}}$ Gongrang Li, ${ }^{\mathrm{C}}$ Tao Wu (D) *a and Dejun Sun ${ }^{\star a}$
}

Crude oil contaminated soil has been widely recognized to constitute a major environmental issue due its adverse effects on human health and ecological safety. The main objective of this study is to explore the possibility of using an ex situ solvent/surfactant washing technique for the remediation of crude oilcontaminated soil. Three organic solvents (methanol, acetone, and toluene) and one surfactant (AES-DOA) were employed to form three kinds of solvent/surfactant systems, and utilized to evaluate the desorption performance of crude oil from soil. Natural soil, crude oil-contaminated soil, and afterremediation soil were characterized by SEM, EDX, FT-IR, and contact angle. The ability of solvent/ surfactant systems to remove crude oil from soil was determined as a function of solvent polarity, mass ratio of solvent to surfactant, temperature, and ionic strength. The removal of crude oil by the toluene/ AES-D-OA system was found to be more effective than the other systems. At a high toluene ratio, more than $97 \%$ of crude oil could be removed from contaminated soil. Crude oil removal efficiency was also found to increase with rising temperature or increasing ionic strength appropriately. Experimental results suggested that, compared to conventional surfactant-aided remediation, the combined utilization of surfactant and solvent achieved superior results for crude oil removal because of their similar compositions and structures in terms of aromaticity and polarity.

Received 4th December 2018

Accepted 7th January 2019

DOI: $10.1039 / c 8 \mathrm{ra09964b}$

rsc.li/rsc-advances has aroused intensive concern. Various biological, physical, and chemical technologies have been investigated and widely utilized for the remediation of contaminated sites. ${ }^{3-7}$ In particular, soil-washing of contaminated sites with extracting solutions has demonstrated great potential for treating not only organic compounds and petroleum hydrocarbons contaminated soils, but also contamination of heavy metals., ${ }^{2,8-12}$ These soil-washing solutions include different types of chemical agents, such as chelating agents, short-chain organic acids, and surfactants. ${ }^{13}$ Among these chemical agents, the addition of a surfactant to an aqueous formulation is a commonly used method for improving the solubility and mobility of hydrophobic organic contaminants. Surfactants represent an amphiphilic molecule, containing simultaneously hydrophilic and hydrophobic moieties in the molecular structure. Surfactants exist in soil-water systems, and could adsorb on the surface of the soil matrix. Generally, the hydrophilic headgroup is considered to enter into the liquid phase, whereas the hydrophobic tail tends to associate with hydrophobic organic pollutants. At low surfactant concentration, the surfactant mainly accumulates at the solid-liquid interface in the form of molecules. With an increase in concentration, the surfactant gradually replaces the interfacial solvent (i.e., water), leading to a low polarity of the solid-liquid interface and decreased interfacial tension. ${ }^{14}$ As the concentration of the surfactant rises above its critical micelle concentration (CMC), surfactant molecules can form micelles 
in the aqueous phase. These micelles could greatly enhance the solubilization of organic contaminants, and thereby increase the rate of mass transfer of the contaminants from the soil phase to the aqueous phase. ${ }^{\mathbf{1 4 , 1 5}}$ Therefore, the utilization of a surfactant as a soil-washing agent appears to constitute a promising strategy for ex situ or in situ remediation. This surfactant-associated soil-washing procedure can remediate contaminated soils mainly based on two mechanisms: (1) dissolution and solubilization of hydrophobic organic contaminants from soils into hydrophobic cores of surfactant micelles; and (2) mobilization induced by reducing interfacial tension. ${ }^{\mathbf{1 3 - 1 5}}$ However, the surfactant-associated soil-washing procedure still faces some major challenges for practical soil remediation, such as low selectivity, long extraction time, and insufficient extraction efficiency. These disadvantages limit practical applications of the surfactantassociated soil-washing procedure. In pragmatic remediation, in addition to surfactants, small amounts of organic solvents are usually employed in extracting low-polar or nonpolar petroleum hydrocarbons from contaminated soils. In this process, their extraction efficiencies were significantly better than those of surfactant alone, and their extraction time was also shorter than those of surfactant alone. ${ }^{\mathbf{1 6 - 1 8}}$ For the above mentioned reasons, the combined utilization of surfactants with other additives (organic solvents) has received substantial focus in recent years.

The aims of this work are: (1) to optimize solvent/surfactantenhanced soil-washing systems for desorbing crude oil from a simulated crude oil-contaminated soil sample. Here, this was investigated by comparing soil-washing performance. In addition, crude oil was used as a model contaminant. Sodium ethoxylated alkyl sulfate (AES), poly(propylene glycol) bis(2aminopropyl ether) (D230) and oleic acid (HOA) were selected to fabricate the AES-D-OA surfactant. Three different polarities of organic solvents were utilized, including methanol, acetone, and toluene, for the preparation of methanol/AES-D-OA-, acetone/AES-D-OA-, and toluene/AES-D-OA-enhanced soil washing systems, respectively, to remove crude oil from the simulated crude oil-contaminated soil sample. The optimal solvent/surfactant-enhanced soil-washing system was chosen in a subsequent set of removal experiments; (2) to evaluate the effects of mass ratio of solvent to surfactant, temperature, and ionic strength on the removal efficiency of crude oil from simulated crude oil-contaminated soil; and (3) to elucidate interactions between the solvent/surfactant system and crude oil-contaminated soil, and the desorption mechanism. All of the obtained information will be useful for further practical applications of the novel solvent/surfactant-enhanced soil-washing system in real contaminated soil remediation.

\section{Materials and methods}

\subsection{Materials}

2.1.1 Chemicals. Methanol (>99.5\%), acetone (>99.5\%), toluene $(>99.5 \%)$, and oleic acid $(>97 \%)$ were purchased from the Sinopharm Chemical Reagent Co., Ltd. (Shanghai, China). Poly(propylene glycol) bis(2-aminopropyl ether) (D230) was purchased from the Sigma-Aldrich Co., Ltd. (U.S.A.). Sodium ethoxylated alkyl sulfate (AES, 70\%) was purchased from the Lvsen Chemical Company (Shandong, China). NaCl (>99\%), and $\mathrm{CaCl}_{2}(>96 \%)$ were purchased from the Aladdin Chemical Regent Co., Ltd. (Shanghai, China). All chemicals were used without further purification, and all chemicals were used as received from the supplier.

2.1.2 Crude oil. The crude oil sample was obtained from the Shengli Oilfield in China, with a water content of less than $0.5 \%$, and density and a viscosity of $938 \mathrm{~kg} \mathrm{~m}^{-3}$ and $985 \mathrm{mPa} \mathrm{s}$ at $50{ }^{\circ} \mathrm{C}$, respectively.

2.1.3 Soil. The clean soil was collected from the Crystal Material Institute site at Shandong University, China. One soil, under-plant, which represents the predominant soil type of the site, was sampled $20 \mathrm{~cm}$ beyond the root zone of the plant and chosen for evaluation in this work. The soil sample was screened via a $2 \mathrm{~mm}$ British Standard Test Sieve to remove coarse fragments and vegetation. The treated soil sample was oven-dried at $100{ }^{\circ} \mathrm{C}$ for $24 \mathrm{~h}$ to a constant weight prior to being stored in an airtight glass container for the soil-washing experiments. The physical and textural characteristics of the soil are given in Table 1.

\subsection{Preparation of surfactant used}

The D-OA superamphiphilic ion pairs were firstly prepared at room temperature as follows: by mixing D230 liquid and oleic acid liquid at a molar ratio of $1: 2$ under magnetic stirring, superamphiphilic ion pairs could be formed via electrostatic interactions. ${ }^{19}$ Then, D-OA superamphiphilic ion pairs were used to fabricate a new surfactant. The preparation of the AESD-OA surfactant was as follows: $0.5 \mathrm{~g}$ AES was added to $99.5 \mathrm{~g}$ aqueous solution, which contained $0.5 \mathrm{~g}$ D-OA superamphiphilic ion pairs. The mass ratio of AES : D-OA $=1: 1$. The mixture was vigorously stirred at room temperature for $15 \mathrm{~min}$ in order to obtain a homogeneous surfactant system.

\subsection{Preparation of crude oil-contaminated soil}

Crude oil-contaminated soil was artificially prepared by mixing an appropriate quantity of crude oil at a known weight with vigorous mechanical stirring, and the mass ratio of crude oil: soil $=1: 5$. The crude oil-contaminated soil was continuously

Table 1 Properties of the selected soil

\begin{tabular}{lllllrr}
\hline Sample & Organic matter (\%) & pH & Silt (\%) & Clay (\%) & Sand (\%) \\
\hline Under-plant & 6.11 & 7.88 & 19.22 & 11.80 & 68.98 & Texture \\
\hline
\end{tabular}

${ }^{a}$ Soil gram size classification is according to international criteria: clay $<0.002 \mathrm{~mm}, 0.002 \mathrm{~mm}<$ silt $<0.02 \mathrm{~mm}$, and $0.02 \mathrm{~mm}<$ sand $<2 \mathrm{~mm}$. 
stirred at room temperature for $3 \mathrm{~h}$ to enable the complete reaction of the crude oil with soil. The simulated crude oilcontaminated soil was stored in a vessel at room temperature for two weeks. Such contaminated soil had an initial crude oil concentration of $167 \mathrm{mg} \mathrm{g}^{-1}(16.7 \%)$, and was directly used in the batch experiments.

\subsection{Desorption experiments}

Batch desorption experiments were performed to examine the extraction of crude oil in a solvent/surfactant-enhanced soilwashing system. Batch desorption experiments were carried out at $\mathrm{pH}=7$, and performed on a TP-350s magnetic heating agitator (MIU, China) with a stirring speed of $350 \mathrm{rpm}$. The effects of solvent polarity, mass ratio of solvent to surfactant (5:1, $3: 1,1: 1,1: 3$, and $1: 5)$, temperature $\left(0{ }^{\circ} \mathrm{C}, 20^{\circ} \mathrm{C}, 40^{\circ} \mathrm{C}\right.$, $60{ }^{\circ} \mathrm{C}$, and $80^{\circ} \mathrm{C}$ ), and ionic strength on crude oil removal efficiency were investigated. For the desorption experiments, $5 \mathrm{~g}$ contaminated soil and $20 \mathrm{~mL}$ soil-washing solution were added into a $100 \mathrm{~mL}$ glass beaker to form a mixture. The $\mathrm{pH}$ of the mixture was adjusted to 7 at a constant ionic strength. The beaker was then placed on a magnetic heating agitator at $60 \pm$ $1{ }^{\circ} \mathrm{C}$ and $350 \mathrm{rpm}$ for $30 \mathrm{~min}$ to achieve desorption equilibrium. To determine the effect of ionic strength, the desorption experiments were conducted by varying types of inorganic salts (e.g., $\mathrm{NaCl}$ and $\mathrm{CaCl}_{2}$ ) and their concentrations $(0.001,0.002$, $0.003,0.004$, and $0.005 \mathrm{~mol} \mathrm{~L}^{-1}$ ) at $60{ }^{\circ} \mathrm{C}$. After desorption equilibrium was achieved, the suspension was centrifuged by a centrifuge (LG 10-2.4 A, Jingli, China) at $3000 \mathrm{rpm}$ for $15 \mathrm{~min}$. Then, the washed soil was dried for $8 \mathrm{~h}$ at $60{ }^{\circ} \mathrm{C}$ with an oven, which could prevent light petroleum hydrocarbons from volatilizing. To quantify the residual amount of crude oil present in the soil, a liquid-solid extraction process was used. This process was carried out in Eppendorf tubes using $\mathrm{CCl}_{4}$ as an extraction agent (contaminated soil : extraction agent $=100 \mathrm{mg}: 25 \mathrm{~mL}$, $\mathrm{w} / \mathrm{v}$ ), and the concentration of the residual oil was analyzed by an infrared spectrometer oil content analyzer (Oil 460, Beijing, China).

\subsection{Characterization}

The structural features and surface morphologies of the natural soil, crude oil contaminated soil, and after-washing soil were analyzed using scanning electron microscopy (SEM, JSM-6700F, JEOL, Japan). An energy-dispersive X-ray spectroscopy (EDX) instrument was attached to the JSM-6700F. Fourier transform infrared spectroscopy (FT-IR, Vector 22, Bruker AXS, Co., Ltd., Germany) of the samples was performed using $\mathrm{KBr}$ pellets in reflectance mode, from $400-4000 \mathrm{~cm}^{-1}$, with a resolution of $2 \mathrm{~cm}^{-1}$. The static contact angle (CA) data were obtained on a Teclis Tracker tensiometer (Teclis Instruments, France) using a sessile water droplet method with $5 \mu \mathrm{L}$ liquid droplets. Prior to the CA test, natural soil, crude oil-contaminated soil, and afterwashing soil particles were affixed onto a steel plate by doublesided adhesive at room temperature. Each reported CA value was obtained by taking more than three measurements at different positions for the same sample.

\section{Results and discussion}

\subsection{Effect of solvent polarity}

To optimize the soil-washing performance of the solvent/ surfactant systems, the appropriate selected solvent is critical. The investigation was performed by comparing the different polarity of solvents, including methanol, acetone, and toluene, in the presence of AES-D-OA surfactant at a fixed mass ratio for desorbing crude oil from a crude oil-contaminated soil sample. It was found that the solvent/surfactant-enhanced systems could significantly improve the overall removal efficiency of crude oil from the soil by $17.5 \%-22 \%$ compared to using surfactant alone, as shown in Fig. 1. Among these solvent/ surfactant systems, the maximum removal efficiency for crude oil was determined to be the toluene/AES-D-OA system, while the minimum removal efficiency for crude oil was found to be the acetone/AES-D-OA system. If the polarity of the solvent constitutes a major factor to affect and control the soil-washing performance, the methanol/AES-D-OA system should be more effective than the acetone/AES-D-OA system and the toluene/ AES-D-OA system for removing crude oil (the order of the polarity of the three organic solvents was methanol $>$ acetone $>$ toluene). However, performance of the methanol/AES-D-OA washing agent was not first, but second. The addition of organic solvents to surfactant solutions can be altered not only by altering the nature of the surfactant, but also by changing the characteristics of the bulk phase, such as aggregation number, micelle ionization degree, micellization of the surfactant, and solubilization capacity of micelle, thereby further affecting the solubilization and mobilization of crude oil by lowering the polarity of the fluid and reducing interfacial tension. ${ }^{\mathbf{2 0 , 2 1}}$

According to the experimental data, the polarity of the solvent was not the sole factor to affect and control the soilwashing performance. Indeed, other than solvent polarity, other interactions between organic solvents and crude oil

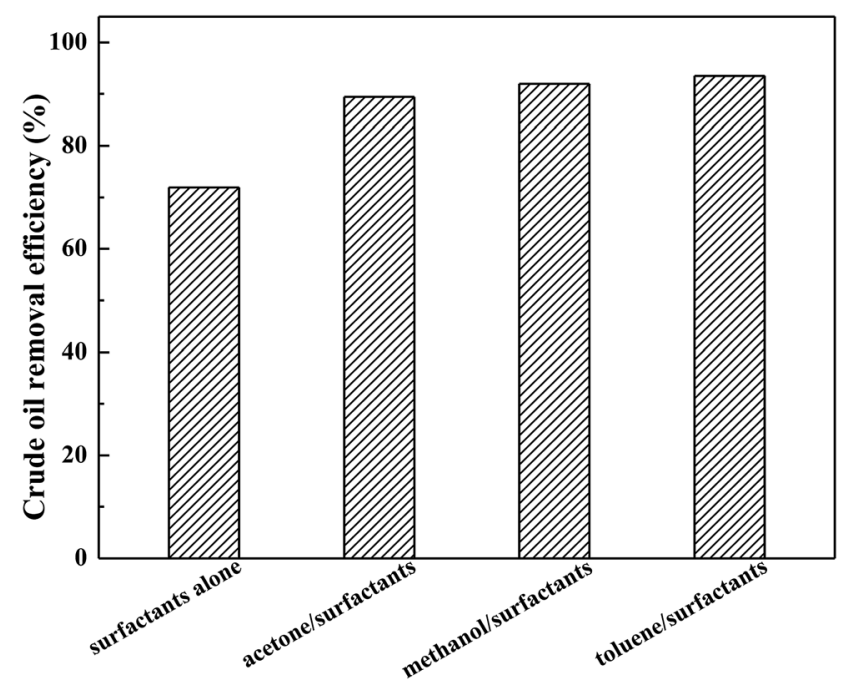

Fig. 1 Effect of polarity of organic solvents on crude oil removal efficiencies (experimental conditions: initial oil concentration = $167 \mathrm{mg} \mathrm{g}^{-1}, \mathrm{pH}=7$, contact time $=30 \mathrm{~min}$ at $60^{\circ} \mathrm{C}$ ). 
components must exist. The toluene molecule is known to comprise an aromatic ring and $\mathrm{CH}_{3}$ group in the molecular structure. On the other hand, petroleum fuels are extremely complex components, with one or more aromatic rings linked with aliphatic chains. With an increase in the aromaticity of a solvent, the toluene/AES-D-OA system is anticipated to provide a strong affinity to crude oil components due to their similar compositions and structures in terms of aromaticity and polarity. Therefore, crude oil components were easily partitioned into a toluene-incorporated AES-D-OA micellar phase. Meanwhile, $\pi-\pi$ interactions and hydrogen bonding could make additional contributions. Therefore, the toluene/AES-DOA washing agent produced the best washing performance. In addition, the presence of toluene in a surfactant solution may reduce the CMC of the AES-D-OA. In this case, a higher concentration of surfactant micelles could be achieved by lowering the CMC of the toluene/AES-D-OA system, which enhances the solubilization and desorption of crude oil from soil. The combined use of surfactants with organic solvents can achieve a synergetic effect for washing agents. Therefore, the toluene/AES-D-OA-enhanced washing agent could be effectively employed to remediate crude oil-contaminated soil.

\subsection{Effect of mass ratio of solvent to surfactant}

In a solvent/surfactant-enhanced washing system, since the soil-washing performance also depends on the mass ratio of solvent to surfactant, five different mass ratios of solvent/ surfactant-enhanced washing systems were used to evaluate this effect. The investigation of the toluene/AES-D-OA system for washing crude oil from contaminated soil was carried out in two sets. In the first set, the concentration of the AES-D-OA was fixed at $1 \mathrm{wt} \%$, and the concentration of toluene was varied from $5 \mathrm{wt} \%$ to $1 \mathrm{wt} \%$ to produce three samples with toluene : AES-DOA mass ratio of $5: 1,3: 1$, and $1: 1$. In the second set, the concentration of toluene was fixed at $1 \mathrm{wt} \%$, and the concentration of the AES-D-OA was varied from $3 \mathrm{wt} \%$ to $5 \mathrm{wt} \%$, resulting in two samples with toluene : AES-D-OA mass ratio of $1: 3$ and $1: 5$. The obtained toluene/AES-D-OA-enhanced washing systems were defined as toluene/AES-D-OA 1, toluene/AES-D-OA 2, toluene/AES-D-OA 3, toluene/AES-D-OA 4, and toluene/AES-D-OA 5, respectively, corresponding to toluene : AES-D-OA mass ratio of $5: 1,3: 1,1: 1,1: 3$, and $1: 5$.

The experimental results are plotted in Fig. 2 . It was observed that the soil-washing performance increased with an increase in toluene concentration, implying that the toluene/AES-D-OA 1 provided the optimal soil-washing performance. Toluene is a very lipophilic amphiphilic additive, which contains hydrophobic (benzene ring) and hydrophilic (polar group) moieties in the molecular structure. The addition of toluene to aqueous AES-D-OA solution may greatly improve the solubilization of AES-D-OA for crude oil. This improvement in the solubility is attributed to the formation of toluene-incorporated surfactant micelles, thereby offering additional interactions between toluene molecules and contaminated soil particles, and promoting contaminant extraction. ${ }^{22-24}$ With an increase in toluene concentration, interactions between solvent molecules

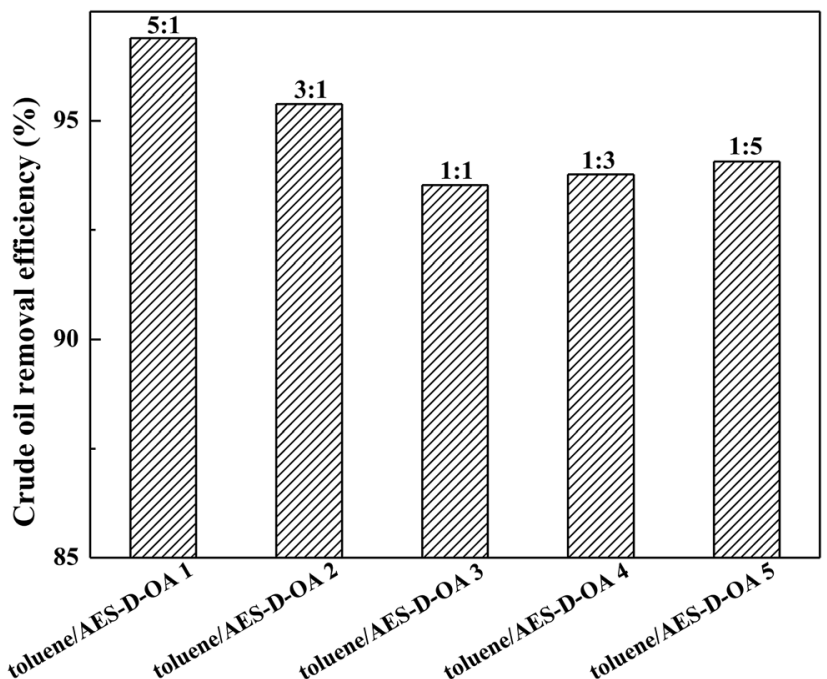

Fig. 2 Effect of mass ratio of toluene to AES-D-OA on crude oil removal efficiencies (experimental conditions: initial oil concentration $=167 \mathrm{mg} \mathrm{g}^{-1}, \mathrm{pH}=7$, contact time $=30 \mathrm{~min}$ at $60^{\circ} \mathrm{C}$ ).

and contaminated soil particles become increasingly stronger. With an addition of toluene to AES-D-OA solution, the micelle size or micelle volume of the surfactant also becomes increasingly large, which augments the ability of the surfactant micellar core to solubilize and mobilize crude oil. ${ }^{25,26}$ Moreover, at higher toluene concentrations, toluene may reduce the viscosity of crude oil and weaken binding to the soil particles, enhancing the mobilization of crude oil in soil, thereby further increasing the mass transfer of pollutants from the solid phase to the aqueous phase. For these reasons, the maximum removal efficiency of crude oil was achieved at toluene : AES-D-OA mass ratio of $5: 1$. Compared to other soil-washing systems, the toluene/AES-D-OA washing agents exhibited superior removal efficiency for crude oil., ${ }^{2,3,27-31}$ As the initial crude oil concentration was $167 \mathrm{mg} \mathrm{g}^{-1}, 94-97 \%$ crude oil could be removed by toluene/AES-D-OA washing agents at $60{ }^{\circ} \mathrm{C}$ (Table 2). Considering remediation cost, toluene/AES-D-OA 3 (mass ratio of toluene : $\mathrm{AES}-\mathrm{D}-\mathrm{OA}=1: 1$ ) was chosen as an effective soil-washing agent for removing crude oil from soil in the subsequent desorption experiments.

\subsection{Effect of temperature}

Temperature constituted a key parameter affecting and controlling the soil-washing performance. The effect of washing temperature on crude oil removal efficiency by using toluene/ AES-D-OA 3 as soil-washing agent was studied in the washing temperature range of $0-80{ }^{\circ} \mathrm{C}$ at a constant $\mathrm{pH}$ value (7.0) and contact time (30 min). As shown in Fig. 3, crude oil removal efficiency increased with rising temperature, indicating that the desorption of crude oil from contaminated soil was endothermic. Overall, increasing temperature helped to obtain better soil-washing performance.

The high soil-washing performance occurring at higher temperature is mainly attributed to: (1) a higher temperature is 
Table 2 Application cases of different washing agents for the remediation of various oil contaminated soil

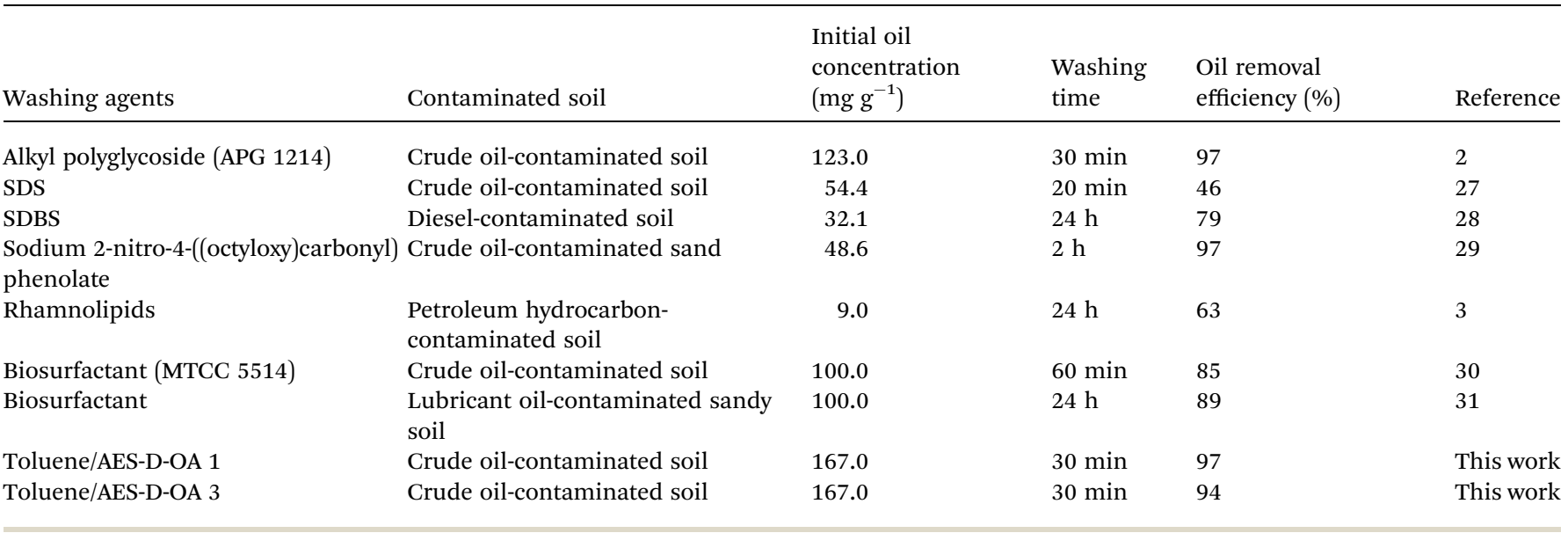

expected to increase the diameter of the micelle and enhance the solubilization of crude oil components; (2) due to the micelle diameter and micelle core volume increasing with rising temperature, ${ }^{4,32,33}$ in which the solubility of crude oil components is increased by partitioning hydrophobic crude oil components into the micelle core, the amount of crude oil dissolved in the micellar phase is increased; (3) an increase in temperature generally results in a decrease in the viscosity of crude oil, which contributes to increasing the mobility of crude oil components in soil; (4) Brownian movement of solvent molecules and the diffusion rate of crude oil components increased with rising temperature. In this case, the crude oil components might be unable to build strong bonds with soil particles, resulting in an increase in the rate of mass transfer from the soil phase to the aqueous phase; and (5) the dominant interaction forces between the crude oil components and soil particles appear to be van der Waals forces. However, it was possible that the van der Waals forces might significantly decrease with increasing temperature, ${ }^{34-38}$ meaning that the

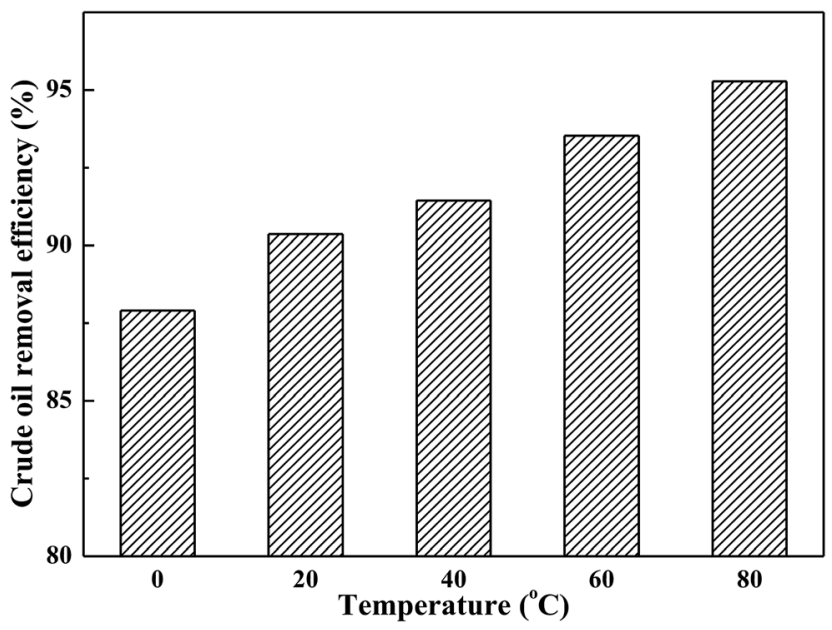

Fig. 3 Effect of temperature on crude oil removal efficiencies by using the toluene/AES-D-OA soil-washing agent (experimental conditions: initial oil concentration $=167 \mathrm{mg} \mathrm{g}^{-1}, \mathrm{pH}=7$, contact time $=30 \mathrm{~min}$ ). interactions between crude oil components and soil particles are weak.

Based on the above analysis, the desorption of crude oil from soil by using the soil-washing technique is a complex process. It involves some interactions, including partitioning, hydrogen bonding, hydrophobic bonding, and $\pi-\pi$ interactions.

\subsection{Effect of ionic strength}

In a surfactant-containing aqueous medium, the formation of a surfactant micelle phase constitutes the major factor in determining soil-washing performance. At a critical micelle concentration, surfactant molecules will start to aggregate and form micelles. When the use of surfactant is above its critical micelle concentration, surfactant micelles can augment the solubilization of low-polar/non-polar organic compounds..$^{39,40}$ The addition of electrolytes (e.g., inorganic salts) to a surfactantcontaining aqueous medium and the binding of counter-ions to the headgroups of surfactants may reduce the electrostatic repulsions between surfactant molecules and dramatically improve the environmental viscosity of polar surfactant headgroups. ${ }^{39-41}$ Therefore, the existence of electrolytes in a surfactant-containing aqueous medium may reduce the CMC of the mixed systems, and consequently the affinity between micelles and crude oil components is enhanced by increasing aggregation number, thereby further promoting solubilization and desorption of contaminants from soil..$^{42,43}$ The present study uses $\mathrm{NaCl}$ and $\mathrm{CaCl}_{2}$ as electrolytes and investigates their effects on soil-washing performance.

The effect of ionic strength on crude oil removal efficiency is shown in Fig. 4. In the salt-free system, the crude oil removal efficiency by toluene/AES-D-OA 3 washing agent reached $94 \%$. In the salt-present system, the crude oil removal efficiency increased with the addition of $\mathrm{NaCl}$ or $\mathrm{CaCl}_{2}$ to toluene/AES-DOA 3 washing solution. Meanwhile, the crude oil removal efficiencies of the NaCl-toluene/AES-D-OA system were higher than those of the $\mathrm{CaCl}_{2}$-toluene/AES-D-OA system. Because the concentration of salts was very low $\left(0<C_{\text {salt }}<0.005 \mathrm{~mol} \mathrm{~L}^{-1}\right)$, the crude oil removal efficiencies of the salt-present system were only slightly higher than those of the salt-free system. It is well 


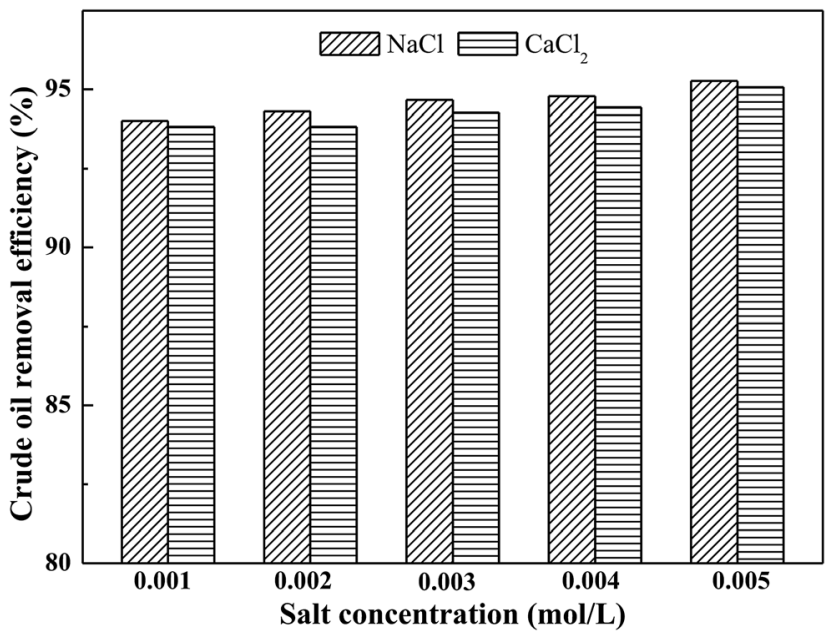

Fig. 4 Effect of salt type and concentration on crude oil removal efficiencies by using the toluene/AES-D-OA soil-washing agent (experimental conditions: initial oil concentration $=167 \mathrm{mg} \mathrm{g}^{-1}, \mathrm{pH}=$ 7 , contact time $=30 \mathrm{~min}$ at $60^{\circ} \mathrm{C}$ )

known that adding electrolytes strongly suppresses the CMC of ionic surfactants. ${ }^{39,42}$ Theoretically, the addition of $\mathrm{NaCl}$ or $\mathrm{CaCl}_{2}$ to toluene/AES-D-OA 3 aqueous solution can reduce the CMC value of AES-D-OA and increase the aggregation number, leading to an increase in crude oil liberation from soil. Moreover, with the addition of $\mathrm{NaCl}$ or $\mathrm{CaCl}_{2}$ to aqueous solution, and the consequent binding of $\mathrm{Na}^{+}$or $\mathrm{Ca}^{2+}$ ions to the headgroups of surfactant molecules, the electrical double layers of headgroups are compressed, and electrostatic repulsion between the ionic headgroups is shielded. Hence, toluene and AES-D-OA tend to form surface active complexes, which favors the adsorption of toluene/AES-D-OA complexes at the crude oilsoil interface, and improves crude oil liberation from soil. Moreover, increasing the salinity concentration of the aqueous phase favors the dissociation reaction, and the small-size solvent molecules are able to penetrate into crude oil components. This results in low viscosity and a more fluid state of the crude oil, thereby enhancing transfer of the crude oil components from the soil phase to the solid-water interface, and into the aqueous phase. ${ }^{44-47}$ The crude oil removal efficiency of the $\mathrm{CaCl}_{2}$-toluene/AES-D-OA system was lower than that of the NaCl-toluene/AES-D-OA system. Compared to $\mathrm{Na}^{+}, \mathrm{Ca}^{2+}$ is a divalent cation and has a stronger effect on reducing electrostatic repulsion between ionic headgroups than does $\mathrm{Na}^{+}$.
However, $\mathrm{CaCl}_{2}$ can strongly interact with AES-D-OA molecules and cause precipitation of the surfactant. This precipitation can cause major losses of surfactant which, in turn, can affect the soil-washing performance of the toluene/AES-D-OA 3 system. ${ }^{48,49}$ In addition, it is possible that $\mathrm{Ca}^{2+}$ ions are hydrated by the water molecules due to their strong potential attraction to water molecules and tend to remain in the aqueous phase. In this case, the combined effect of solvent and surfactant on the solubilization of crude oil components could be reduced by lowering the surfactant concentration. ${ }^{50,51}$ Increasing the cationic state of the electrolytes was found to slightly decrease the liberation of crude oil from the soil surface.

The presence of salts slightly improved soil-washing performance. This phenomenon can be explained by: (1) the screening effect of the electrical double layer by the salt; and (2) the decreasing electrostatic repulsion between anionic headgroups of AES-D-OA, thus increasing the surface activity of complexes.

\subsection{Characterization}

3.5.1 SEM and EDX analysis. The morphological and structural features of natural soil, crude oil-contaminated soil, and after-remediation soil were characterized by SEM. From the SEM image (Fig. 5(a)) of natural soil, it can be seen that the soil sample exhibits a massive and agglomerated morphology with a size range between 5 and $20 \mu \mathrm{m}$. According to the SEM image (Fig. 5(b)), crude oil is strongly bound to the soil particles via van der Waals forces and hydrogen bonding. Consequently, the morphology of the crude oil-contaminated soil exhibits a 'dense package' flake-like structure. As shown in Fig. 5(c), the remediation of soil shows a great improvement in dispersion degree. The microscopic surface of remediated soil with toluene/AES-DOA system is similar to surface of the natural soil and displays a color change from black to yellow-brown, indicating that crude oil is removed from the soil.

In addition, the surface element compositions of natural soil, crude oil-contaminated soil, and after-remediation soil were analyzed by EDX. As shown in Fig. 6(a), EDX quantitative microanalysis indicated the presence of $\mathrm{Si}, \mathrm{Ti}, \mathrm{Mg}, \mathrm{Al}, \mathrm{Ca}, \mathrm{Fe}, \mathrm{K}$, $\mathrm{O}$, and $\mathrm{C}$ in the natural soil. The EDX analysis results suggested that $\mathrm{C}, \mathrm{O}, \mathrm{S}, \mathrm{Si}, \mathrm{Al}, \mathrm{Mg}, \mathrm{Ca}$, and $\mathrm{K}$ were uniformly distributed in the crude oil-contaminated soil (Fig. 6(b)). The EDX analysis for the remediation of soil confirmed the crude oil liberation from the soil and element composition, in which $\mathrm{Si}, \mathrm{Al}, \mathrm{C}$, and $\mathrm{O}$ were
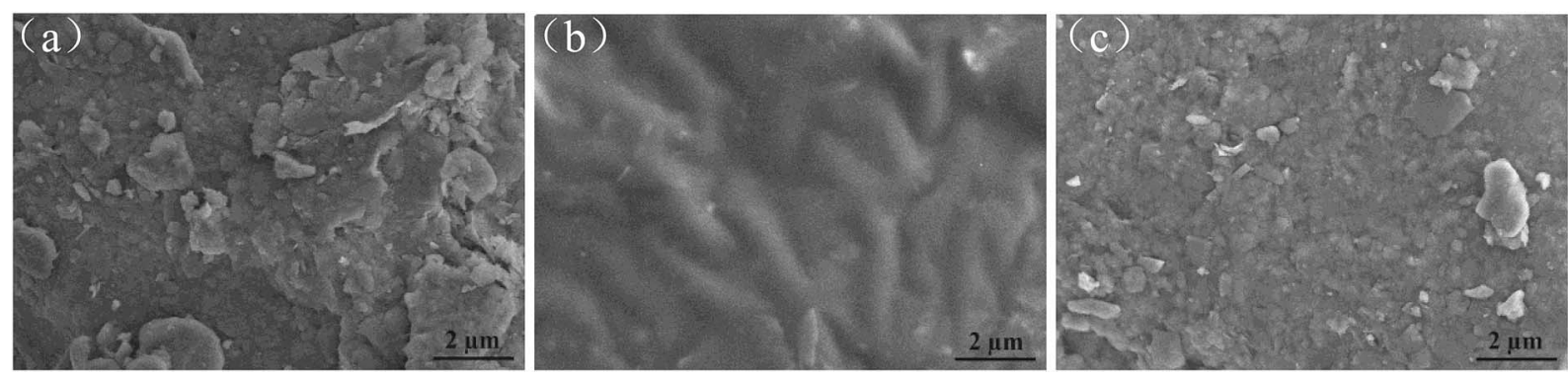

Fig. 5 SEM images of (a) natural soil, (b) crude oil-contaminated soil, and (c) after-remediation soil. 

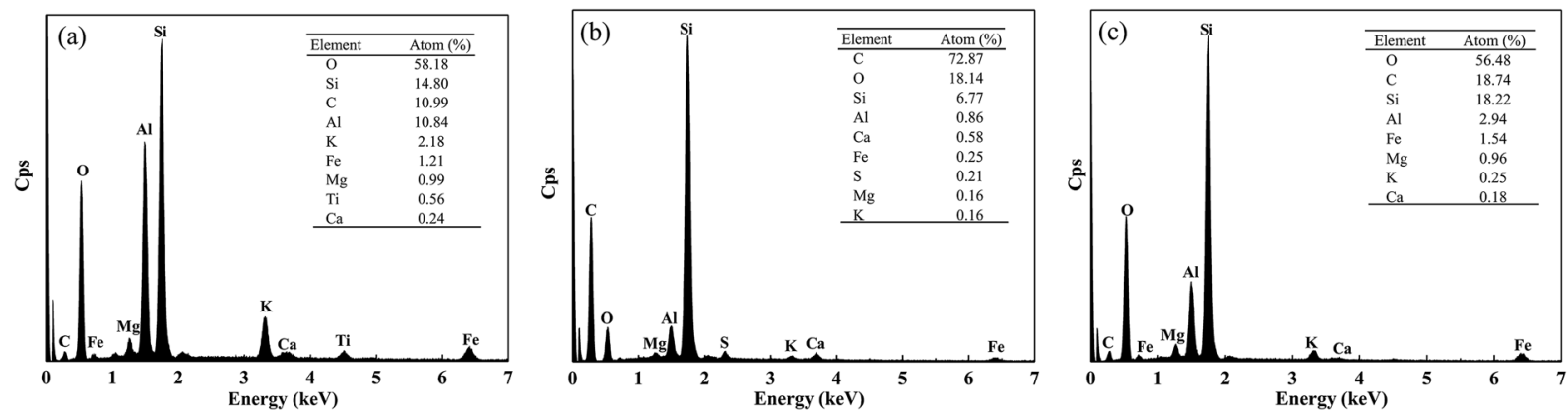

Fig. 6 EDX analysis of (a) natural soil, (b) crude oil-contaminated soil, and (c) after-remediation soil.

detected as the major constituents (Fig. 6(c)). In some way, soilwashing performance was also characterized by the change of carbon content because the vast majority of crude oil is hydrocarbon. The content of carbon was $10.99 \%$, which might be organic matter in natural soil (Fig. 6(a)). In Fig. 6(b), the percentage of carbon was very high $(72.87 \%)$ due to the large amount of crude oil in contaminated soil. However, the content of carbon had a low percentage of $18.74 \%$, which indicated that crude oil was almost removed from soil and had a little residual oil in soil.

3.5.2 FT-IR. FT-IR technology is an essential and useful tool to obtain information about surface functional groups and chemical binding behavior. ${ }^{52}$ The FT-IR spectra of natural soil, crude oil-contaminated soil, and after-remediation soil are shown in Fig. 7(a)-(c). For natural soil, the bands in the FT-IR spectrum can be summarized as follows: (1) a strong broad band observed at $3624 \mathrm{~cm}^{-1}$ is attributed to the free $\mathrm{O}-\mathrm{H}$; (2) the adsorption band that appeared at $3430 \mathrm{~cm}^{-1}$ is assigned to hydrogen bonded $\mathrm{O}-\mathrm{H} ;{ }^{53,54}$ (3) the band at $1632 \mathrm{~cm}^{-1}$ is due to $\nu_{\mathrm{H}-\mathrm{O}-\mathrm{H}}$ bonding vibration; ${ }^{55}$ (4) the adsorption band at $1436 \mathrm{~cm}^{-1}$ is attributed to the vibration of $\mathrm{CO}_{3}{ }^{2-}\left(\nu_{3}\right) ;{ }^{56}(5)$ the $\mathrm{Si}-\mathrm{O}-\mathrm{Si}$ band is observed at $1027 \mathrm{~cm}^{-1}$ as a result of the $\mathrm{Si}-\mathrm{O}$ vibration; and (6) the bands at $780,688,533$, and $469 \mathrm{~cm}^{-1}$ are

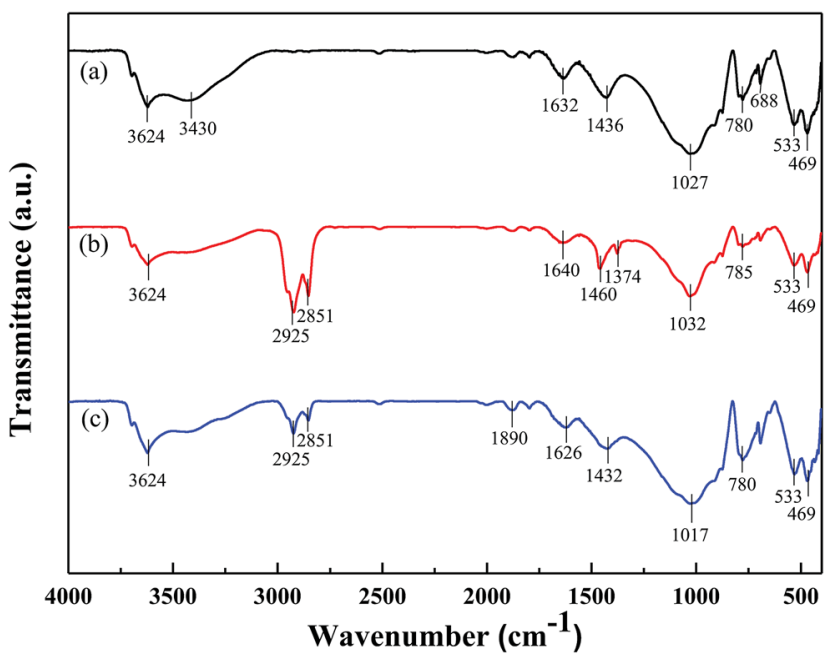

Fig. 7 FT-IR spectra of (a) natural soil, (b) crude oil-contaminated soil, and (c) after-remediation soil. attributed to $\mathrm{Mg}-\mathrm{O}$ and Al-O lattice vibrations. ${ }^{56,57}$ For the FT-IR spectra of crude oil-contaminated soil, three main regions are identified: (1) $2925 \mathrm{~cm}^{-1}$ and $2851 \mathrm{~cm}^{-1}$ : attributed to $\mathrm{C}-\mathrm{H}$ asymmetric and symmetric stretching vibrations of $\mathrm{CH}_{2}$ and $\mathrm{CH}_{3}$ groups; (2) $1460 \mathrm{~cm}^{-1}$ and $1374 \mathrm{~cm}^{-1}$ : attributed to $\mathrm{C}-\mathrm{C}$ stretching vibration; and (3) $900 \mathrm{~cm}^{-1}$ and $700 \mathrm{~cm}^{-1}$ : attributed to the characteristic peaks of aromatic structure. ${ }^{58,59}$ In addition, the peak observed at $1032 \mathrm{~cm}^{-1}$ was assigned to the $\mathrm{C}-\mathrm{O}$ stretching vibration for the crude oil, indicating the presence of alcohol and carbonyl groups. ${ }^{59,60}$ Furthermore, the peaks belonging to the soil remained at almost constant positions. For the remediation of soil using the toluene/AES-D-OA washing agent, the band at $1626 \mathrm{~cm}^{-1}$ was associated with the $\mathrm{N}-\mathrm{H}$ stretching vibration, indicating the presence of residual AES-DOA surfactant in soil during the washing process. Moreover, the C-C stretching vibration shifted to a lower wavelength (from $1460 \mathrm{~cm}^{-1}$ to $1432 \mathrm{~cm}^{-1}$ ), and the $\mathrm{C}-\mathrm{O}$ stretching mode also shifted to a lower wavelength (from $1032 \mathrm{~cm}^{-1}$ to $1017 \mathrm{~cm}^{-1}$ ). Meanwhile, the intensity of $\mathrm{C}-\mathrm{H}$ asymmetric $\left(2925 \mathrm{~cm}^{-1}\right)$ and symmetric $\left(2851 \mathrm{~cm}^{-1}\right)$ stretching vibrations became significantly weak compared to crude oil-contaminated soil. These changes indicated that crude oil was effectively removed from the soil by the soil-washing process.

3.5.3 Wettability. Wettability is an essential and important property of a solid surface, which determines wetting behavior when a liquid droplet comes into contact with a solid surface. ${ }^{52}$ The wettability of a solid surface with a liquid droplet is controlled by its chemical properties and surface microstructures. ${ }^{61} \mathrm{~A}$ surface is hydrophilic if it has a water contact angle (CA) of $<10^{\circ}$, and a surface is hydrophobic if it has a water CA above $90^{\circ} .{ }^{62} \mathrm{~A}$ CA measurement technique could be utilized to determine the wetting behavior of a solid surface. To avoid the measuring time from being too long or too short, the reported water CA values of natural soil, crude oil-contaminated soil, and after-remediation soil were obtained as the water droplet came into contact with the soil surface at $5 \mathrm{~s}$. An approximate $5 \mu \mathrm{L}$ water droplet was placed on the surface of natural soil. It then spreads quickly and a near zero CA is reached (Fig. 8(a)), indicating that the natural soil is highly hydrophilic. When a water droplet with the same volume is placed on the surface of crude oil-contaminated soil, the soil sample gives water CA of $113^{\circ}$, which implies that the crude oil components are strongly bonded to soil particles, and the surface of crude oil- 

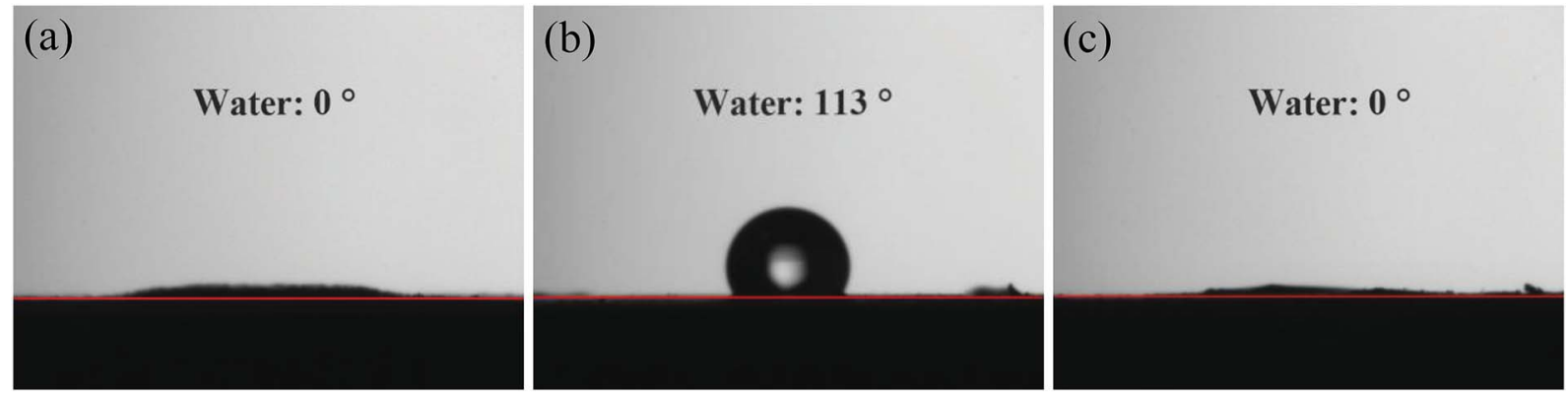

Fig. 8 Water CAs of (a) natural soil, (b) crude oil-contaminated soil, and (c) after-remediation soil.

contaminated soil is difficult to be wetted by water (Fig. 8(b)). After the remediation of soil by using the toluene/AES-D-OA washing agent, a water CA of $0^{\circ}$ is obtained (Fig. 8(c)), suggesting that the crude oil has been successfully removed from the contaminated soil.

\subsection{Remediation mechanism}

Remediation of crude oil-contaminated soil mainly involves the surfactant's solubilization principle. In the present study, using the concentration of the surfactant in the soil-washing agents at above its CMC, surfactant molecules can form micelles in aqueous solution. Once the soil-washing agent comes into contact with crude oil-contaminated soil, these micelles could greatly enhance the solubilization of organic contaminants, and thereby the hydrophobic crude oil is considered to solubilize into hydrophobic cores of the surfactant micelles. After a complete reaction of the soil-washing agent with crude oilcontaminated soil, centrifugation technology can be utilized to separate the solid phase and the aqueous phase. In this case, washing solution in which containing crude oil could be separated from soil and achieved the purpose of remediation. Small organic solvents are usually employed in extracting low-polar or non-polar petroleum hydrocarbons from contaminated soil. In this process, the addition of organic solvents to surfactant solution can be altered not only by altering the nature of the surfactants, but also by changing the characteristics of the bulk phase, such as micelles ionization degree, aggregation number, micelle core volume, and solubilization capacity of micelle, thereby further affecting the overall solubilization of crude oil. At the same time, organic solvents, especially aromatic organic solvents, were able to penetrate between heavy petroleum molecules and swell their branches, ${ }^{\mathbf{4}}$ which enhanced the fluidity of crude oil. As a result, viscosity of crude oil decreased and the desorption of crude oil from contaminated soil was accelerated.

\section{Conclusions}

The purpose of the present study was to evaluate the addition of an organic solvent (i.e., methanol, acetone, or toluene) as a combined approach towards the removal of crude oil components, since solubilization, extraction, and desorption of hydrophobic crude oil components constitutes one of the most important tasks in soil remediation. Based on the experimental data, solvent/surfactant soil-washing systems have been proven to be effective to solubilize and extract crude oil components from soil. It is noted that a synergistic effect of solvent and surfactant could be achieved by forming solvent-incorporated surfactant micelles, thereby promoting contaminant solubilization, extraction, and desorption. In other words, the micellesolubilized solvent has a positive effect in promoting the partition process and improving the driving forces for removing hydrophobic crude oil components. However, our results also suggest that the selection of an appropriate solvent is critical to effectively complex and solubilize the crude oil from soil. Furthermore, an optimal matching of mass ratio between solvent and surfactant is also requisite for an integrated consideration of both remediation cost and crude oil removal. Therefore, various parameters, including solvent polarity, mass ratio of solvent to surfactant, temperature, and ionic strength, should be taken into account. The results of this study demonstrate that the toluene/AES-D-OA system is expected to provide a strong affinity to crude oil components because of their similar compositions and structures in terms of aromaticity and polarity. ${ }^{44}$ At a high toluene ratio, more than $97 \%$ of crude oil could be removed. Overall, increasing temperature and ionic strength contributed to removing crude oil from soil. In addition, SEM images, EDX analysis, FT-IR analysis, and CA measurements confirmed the desorption of crude oil from soil by using the toluene/AES-D-OA system. This study showed that the toluene/AES-D-OA system could be applied as an efficient soil-washing agent to separate and desorb highly hydrophobic crude oil components from soil, which constitutes a needed application in soil remediation.

\section{Conflicts of interest}

There are no conflicts to declare.

\section{Acknowledgements}

This work was supported by the National Natural Science Foundation of China (grant no. 21333005 and 21677087) and the National Science and Technology Major Project of China (grant no. 2016ZX05040-005). The authors express gratitude to Jake Carpenter from UCLA for linguistic assistance. 


\section{References}

1 W. Chu and C. Y. Kwan, Chemosphere, 2003, 53, 9-15.

2 M. Han, G. Ji and J. Ni, Chemosphere, 2009, 76, 579-586.

3 C.-C. Lai, Y.-C. Huang, Y.-H. Wei and J.-S. Chang, J. Hazard. Mater., 2009, 167, 609-614.

4 S. Paria, Adv. Colloid Interface Sci., 2008, 138, 24-58.

5 M. S. Kuyukina, I. B. Ivshina, S. O. Makarov, L. V. Litvinenko, C. J. Cunningham and J. C. Philp, Environ. Int. , 2005, 31, 155161.

6 P.-C. Zhang, J. L. Krumhansl and P. V. Brady, SSSA Special Publication, Geochemistry of Soil Radionuclides, Soil Science Society of America, 2002, pp. 1-20.

7 X. Ma, Y. Luan, S. Liu, Y. Liu and C. Xia, $R S C A d v .$, 2015, 5, 42597-42602.

8 S. Lamichhane, K. C. Bal Krishna and R. Sarukkalige, J. Environ. Manage., 2017, 199, 46-61.

9 R. J. Abumaizar and E. H. Smith, J. Hazard. Mater., 1999, 70, 71-86.

10 R. Anderson, E. Rasor and F. Van Ryn, J. Hazard. Mater., 1999, 66, 89-98.

11 Y. Wei, X. Liang, L. Tong, C. Guo and Z. Dang, Colloids Surf., A, 2015, 468, 211-218.

12 J. Tang, J. He, T. Liu and X. Xin, $R S C$ Adv., 2017, 7, 3338533401.

13 K. Urum, T. Pekdemir and M. opur, J. Colloid Interface Sci., 2004, 276, 456-464.

14 C. N. Mulligan, R. N. Yong and B. F. Gibbs, Eng. Geol., 2001, 60, 371-380.

15 X. Mao, R. Jiang, W. Xiao and J. Yu, J. Hazard. Mater., 2015, 285, 419-435.

16 L. Ramos, J. Tabera, L. M. Hernández and M. J. González, Anal. Chim. Acta, 1998, 376, 313-323.

17 W. Chu and C. Y. Kwan, Water Res., 2002, 36, 2187-2194.

18 A. Hubert, K.-D. Wenzel, M. Manz, L. Weissflog, W. Engewald and G. Schüürmann, Anal. Chem., 2000, 72, 1294-1300.

19 P. Xu, Z. Wang, Z. Xu, J. Hao and D. Sun, J. Colloid Interface Sci., 2016, 480, 198-204.

20 A. Rodríguez, M. Del Mar Graciani, M. Muñoz, I. Robina and M. L. Moyá, Langmuir, 2006, 22, 9519-9525.

21 R. Nagarajan and C.-C. Wang, Langmuir, 2000, 16, 52425251.

22 A. Graciaa, J. Lachaise, C. Cucuphat, M. Bourrel and J. L. Salager, Langmuir, 1993, 9, 669-672.

23 A. Graciaa, J. Lachaise, C. Cucuphat, M. Bourrel and J. L. Salager, Langmuir, 1993, 9, 3371-3374.

24 Y. G. Mishael and P. L. Dubin, Langmuir, 2005, 21, 98039808.

25 C. A. Ramsburg and K. D. Pennell, Environ. Sci. Technol., 2002, 36, 2082-2087.

26 C. T. Jafvert, L. V. H. Patricia and J. K. Heath, Water Res., 1994, 28, 1009-1017.

27 K. Urum, S. Grigson, T. Pekdemir and S. Mcmenamy, Chemosphere, 2006, 62, 1403-1410.
28 A. Hernández-Espriú, E. Sánchez-León, P. Martínez-Santos and L. G. Torres, J. Soils Sediments, 2013, 13, 152-165.

29 E. Ceschia, J. R. Harjani, C. Liang, Z. Ghoshouni, T. Andrea, R. S. Brown and P. G. Jessop, RSC Adv., 2014, 4, 4638-4645.

30 V. Kavitha, A. B. Mandal and A. Gnanamani, Int. Biodeterior. Biodegrad., 2014, 94, 24-30.

31 R. M. Jain, K. Mody, A. Mishra and B. Jha, Carbohydr. Polym., 2012, 89, 1110-1116.

32 M. J. Schick, Nonionic Surfactants; Physical Chemistry, 1987, vol. 23.

33 D. Myers, Surfactant science and technology, VCH, 1992.

34 B. Zhang, Z. Dong, D. Sun, T. Wu and Y. Li, J. Ind. Eng. Chem., 2017, 49, 208-218.

35 V. V. Naik and S. Vasudevan, J. Phys. Chem. C, 2011, 115, 8221-8232.

36 Y.-T. Fu and H. Heinz, Chem. Mater., 2010, 22, 1595-1605.

37 H. Heinz, R. A. Vaia and B. L. Farmer, J. Chem. Phys., 2006, 124, 224713.

38 M. Ahmaruzzaman and D. K. Sharma, J. Colloid Interface Sci., 2005, 287, 14-24.

39 J. C. Hoskins and A. D. King, J. Colloid Interface Sci., 1981, 82, 264-267.

40 Q. Xu, M. Nakajima, S. Ichikawa, N. Nakamura, P. Roy, H. Okadome and T. Shiina, J. Colloid Interface Sci., 2009, 332, 208-214.

41 A. K. Chattopadhyay, L. Ghaicha, S. G. Oh and D. O. Shah, J. Phys. Chem., 1992, 96, 6509-6513.

42 S. Deshpande, B. J. Shiau, D. Wade, D. A. Sabatini and J. H. Harwell, Water Res., 1999, 33, 351-360.

43 J. Lee, J.-S. Yang, H.-J. Kim, K. Baek and J.-W. Yang, Desalination, 2005, 184, 395-407.

44 L. He, F. Lin, X. Li, H. Sui and Z. Xu, Chem. Soc. Rev., 2015, 44, 5446-5494.

45 S. Basu, K. Nandakumar, S. Lawrence and J. Masliyah, Fuel, 2004, 83, 17-22.

46 Y. Wang, W. Jia, M. Ding, H. Yang, B. Hu and S. Ren, Energy Fuels, 2012, 26, 1019-1027.

47 F. Lin, L. He, B. Primkulov and Z. Xu, J. Phys. Chem. C, 2014, 118, 13552-13562.

48 D. A. Edwards, R. G. Luthy and Z. Liu, Environ. Sci. Technol., 1991, 25, 127-133.

49 J. D. Rouse, D. A. Sabatini and J. H. Harwell, Environ. Sci. Technol., 1993, 27, 2072-2078.

50 J. Cruz-Hernandez, R. Islas-Juarez, C. Perez-Rosales, S. RivasGomez, A. Pineda-Munoz and J. A. Gonzalez-Guevara, in SPE Latin American and Caribbean Petroleum Engineering Conference Society of Petroleum Engineers, Buenos Aires, Argentina, 2001.

51 N. Yekeen, M. A. Manan, A. K. Idris and A. M. Samin, J. Pet. Sci. Eng., 2017, 149, 612-622.

52 Y. Li, M. Wang, D. Sun, Y. Li and T. Wu, Appl. Clay Sci., 2018, 157, 227-236.

53 L. Zhang, B. Zhang, T. Wu, D. Sun and Y. Li, Colloids Surf., A, 2015, 484, 118-129.

54 U. F. Alkaram, A. A. Mukhlis and A. H. Al-Dujaili, J. Hazard. Mater., 2009, 169, 324-332. 
55 R.-X. Wang, T. Wen, X.-L. Wu and A.-W. Xu, RSC Adv., 2014, 4, 21802-21809.

56 M. Z. B. Hussein and T. K. Hwa, J. Nanopart. Res., 2000, 2, 293-298.

57 N. Roostaei and F. H. Tezel, J. Environ. Manage., 2004, 70, 157-164.

58 J. Hoffmann, C. U. Jensen and L. A. Rosendahl, Fuel, 2016, 165, 526-535.
59 K. a. P. Colati, G. P. Dalmaschio, E. V. R. De Castro, A. O. Gomes, B. G. Vaz and W. Romão, Fuel, 2013, 108, 647-655.

60 Q. Wang, D. Cui, P. Wang, J. Bai, Z. Wang and B. Liu, Fuel, 2018, 211, 341-352.

61 S. Nishimoto and B. Bhushan, RSC Adv., 2013, 3, 671-690.

62 J. Drelich, E. Chibowski, D. D. Meng and K. Terpilowski, Soft Matter, 2011, 7, 9804-9828. 